\title{
BMJ Restless legs syndrome and all-cause Open mortality in four prospective cohort studies
}

\author{
András Szentkirályi, ${ }^{1,2}$ Anke C Winter, ${ }^{3}$ Markus Schürks, ${ }^{4}$ Henry Völzke, ${ }^{5}$ \\ Wolfgang Hoffmann, ${ }^{5,6}$ Julie E Buring, ${ }^{3} \mathrm{~J}$ Michael Gaziano, ${ }^{3,7,8}$ Tobias Kurth, ${ }^{3,9,10}$ \\ Klaus Berger ${ }^{1}$
}

To cite: Szentkirályi A, Winter AC, Schürks $M$, et al. Restless legs syndrome and all-cause mortality in four prospective cohort studies. BMJ Open 2012;2:e001652. doi:10.1136/bmjopen-2012001652

- Prepublication history for this paper are available online. To view these files please visit the journal online (http://dx.doi.org/10.1136/ bmjopen-2012-001652).

AS and ACW contributed equally to the work.

TK and KB jointly directed the work.

Received 12 June 2012 Accepted 19 September 2012

This final article is available for use under the terms of the Creative Commons Attribution Non-Commercial 2.0 Licence; see http://bmjopen.bmj.com

For numbered affiliations see end of article

Correspondence to Dr András Szentkirályi; szentkir@uni-muenster.de

\section{ABSTRACT}

Objectives: To evaluate the association between restless legs syndrome (RLS) and all-cause mortality. Design: Four prospective cohort studies.

Setting: The Dortmund Health Study (DHS) and the Study of Health in Pomerania (SHIP) from Germany. The Women's Health Study (WHS) and the Physicians' Health Study (PHS) from the USA.

Participants: In DHS: a random sample ( $n=1299)$ from the population of Dortmund; in SHIP: a sample $(n=4$ 291) from residents living in West Pomerania were drawn by multistage random sampling design; in WHS: female healthcare professionals $(n=31370)$; in PHS: male physicians ( $n=22$ 926)

Main outcome measures: All-cause mortality.

Results: The prevalence of RLS ranged between $7.4 \%$ and $11.9 \%$ at baseline. During follow-up (ranging between 6 and 11 years) RLS was not associated with increased risk of all-cause mortality in any of the four cohorts. The multivariable-adjusted HRs $(95 \% \mathrm{Cl})$ for all-cause mortality ranged from 0.21 (0.03 to 1.53$)$ to 1.07 (0.93 to 1.23) across the four studies. The HRs for all-cause mortality did not differ according to gender.

Conclusions: In these four independently conducted large prospective cohort studies from Germany and the USA, RLS did not increase the risk of all-cause mortality. These findings do not support the hypothesis that RLS is a risk factor for mortality of any cause.

\section{INTRODUCTION}

Restless legs syndrome (RLS) is a common sleep-related movement disorder affecting about $7-10 \%$ of the general population in western countries. ${ }^{1}$ RLS is characterised by an urge to move the legs, typically accompanied by uncomfortable leg sensations and both feelings are relieved by leg movement. Symptoms emerge during inactivity and they are worst in evening or night hours. The prevalence and incidence of RLS increase with age, and women are more commonly

\section{ARTICLE SUMMARY}

\section{Article focus}

- The aim of this study is to evaluate the association between restless legs syndrome (RLS) and all cause mortality in four independent prospective cohort studies.

\section{Key messages}

- Results of our study do not indicate that RLS is associated with an increased risk for all-cause mortality.

- The risk for all-cause mortality did not differ according to gender.

- RLS should not be considered a risk factor for all-cause mortality.

Strengths and limitations of this study

- Strengths of the study are the inclusion of four different cohorts, the prospective study design, the standardised RLS assessment according to four minimal diagnostic criteria.

- Limitations of the study are as follows: RLS information was self-reported and misclassification of cases was possible; no information on frequency, severity and duration of RLS symptoms was available; the cohorts consist of a predominately white population which may limit the generalisability of the results to other cohorts.

affected. $^{1}{ }^{2}$ The exact pathophysiological pathways of RLS remain unknown; the impairment of the central dopaminergic system and iron deficiency in the brain have a key importance, but genetic risk variants have also been identified. ${ }^{3}$ RLS is frequently associated with insomnia, impaired quality of life and depression. ${ }^{4-6}$

In recent years, results from several crosssectional studies suggested an association between RLS and chronic diseases, especially cardiovascular disorders, such as hypertension, stroke and myocardial infarction. ${ }^{7-11}$ These are frequent disorders in the elderly and associated with a clearly increased risk of mortality. However, the relation between RLS 
and mortality is unclear since prospective studies using the minimal criteria for RLS are lacking so far. The few existing studies either examined clinic-based populations or did not apply the four minimal diagnostic criteria for RLS, which were first introduced in $1995 .{ }^{12}$ RLS was reported to be a marker of higher mortality among patients with chronic renal disease, ${ }^{13-15}$ a condition known to predispose to RLS. Among elderly community dwelling female residents RLS was found to be related to mortality. ${ }^{16}$ Similarly, in a middle-aged sample from the general population RLS was a risk factor for mortality only in women according to a 20-year follow-up study. ${ }^{17}$ Since case classification relies entirely on selfreported symptoms and disease characteristics, application of the minimal criteria for RLS, defined by the International RLS Study Group, is important to allow standardised RLS assessment across populations.

Despite the high prevalence and subjective burden of RLS, it often remains unrecognised in primary care. Given the high frequency of RLS in the general population, an association between RLS and all-cause mortality would have a substantial impact on the population level. Once diagnosed, RLS can be successfully treated in many cases. Thus, the evaluation of RLS as a novel modifiable mortality risk factor has considerable public health implications, and may result in more routine screening and monitoring of RLS.

Therefore, the aim of this study was to investigate whether RLS, assessed by the minimal criteria, is associated with an increased risk of all-cause mortality in four large, independent cohort studies, two from Germany and two from the USA.

\section{METHODS}

The following four prospective cohort studies were included in the analyses. All four studies applied the minimal diagnostic criteria for RLS by using the same short set of standardised questions, ${ }^{12}$ either in German or in English.

\section{DORTMUND HEALTH STUDY}

Primary aim of the Dortmund Health Study (DHS) was to determine the frequency of headache disorders, cardiovascular and other chronic diseases, and behaviourdependent risk factors in the population of the city of Dortmund in the western part of Germany. ${ }^{18}$ From a total population of 591000 a random sample of 3820 persons aged 25-75 years was drawn from the municipal registry. Of those sampled, 395 persons were excluded because they had moved out of the study area, died or did not have sufficient knowledge of the German language leaving 3425 persons, who were eligible and invited to participate in a personal interview at the DHS study centre. If personal participation at the centre was impossible, a questionnaire with a subset of the otherwise identical questions was mailed to the participants. The overall response at baseline was $66.9 \%$, yielding
2291 participants (1312 with interview and 979 with questionnaire). RLS assessment at baseline was restricted to interviewed participants only, because the respective questions were not included in the questionnaire due to the reduced space available. Vital status of 13 subjects could not be identified during the follow-up. Eight had moved outside Germany and were not traceable, three of the remaining five had foreign nationalities and were likely to have moved abroad, and two were untraceable. Therefore, the analyses included the data of 1299 participants from DHS.

\section{STUDY OF HEALTH IN POMERANIA}

The Study of Health in Pomerania (SHIP) is an ongoing population-based study comprising 3 cities and 29 communities in the rural area close to the Baltic Sea (West Pomerania). It was designed to assess a broad range of health and quality-of-life indicators in the north-east region of Germany after the German reunification. ${ }^{19}$ From the total population of 212157 residents living in the study area in 1995, a sample of 7008 men and women aged 20-79 stratified by 5-year age groups was drawn using a multistage random sampling design. The final number of subjects participating in the study was 4308 (response $68.8 \%$ ). The baseline examination was conducted from 1997 to 2001, combining an interview, medical and dental examinations performed in one single visit in the study centre. RLS data at baseline were available from 4291 participants.

\section{WOMEN'S HEALTH STUDY}

The Women's Health Study (WHS) was a randomised, placebo-controlled trial designed to test the risks and benefits of low-dose aspirin (100 mg every other day) and vitamin E (600 IU every other day) in the primary prevention of cardiovascular diseases (CVD) and cancer among apparently healthy women. The design and methods of the WHS have been described in detail previously. ${ }^{20}{ }^{21}$ Briefly, a total of 39876 US female healthcare professionals aged 45 years or older at study entry (1992-1995) without a history of CVD, cancer or other major illnesses were randomly assigned to receive active aspirin, active vitamin E, both active agents, or both placebos. Baseline information was self-reported and collected by a mailed questionnaire that asked about many cardiovascular risk factors and lifestyle variables. Twice in the first year and yearly thereafter, participants were sent follow-up questionnaires asking about study outcomes and other information during the study period. After the trial's termination in March 2004, the women who were still alive and willing to participate entered an observational follow-up. The return date of the 108-month questionnaire containing questions on RLS was defined as new baseline for this analysis. Of the 33092 women in active follow-up at 108 months, we excluded 1722 women with missing RLS information, leaving a total of 31370 women for this analysis. 


\section{PHYSICIANS' HEALTH STUDY}

The Physicians' Health Study I (PHS I) was a randomised, double-blind, placebo-controlled trial to test the benefits and risks of low-dose aspirin (325 mg) and $\beta$-carotene $(50 \mathrm{mg})$ in the primary prevention of CVD and cancer among 22071 apparently healthy male physicians aged 40-84 years at baseline in $1982 .{ }^{22}$ Baseline information was self-reported and collected by means of a mailed questionnaire that asked about many cardiovascular risk factors and life style variables. Every 6 months in the first year and yearly thereafter, follow-up questionnaires were sent to the participants. Since the trials' termination in 1995, the men are continued to be followed either on an observational basis or as part of the PHS II.

Using methods successfully developed in the PHS I, the PHS II was launched in $1997 .{ }^{23}$ The PHS II is an ongoing randomised, double-blind, placebo-controlled trial to test the effects of vitamin C (500 mg), vitamin E (400 IU), $\beta$-carotene $(50 \mathrm{mg})$, and a daily multivitamin (Centrum Silver) in the prevention of total and prostate cancer, CVD and age-related eye disease among 14641 US male physicians aged 55 years and older, including a total of 7641 PHS I participants who were willing and eligible to enter the PHS II. Baseline information was self-reported and follow-up information was collected annually by mailed questionnaires. For the purpose of this analysis, we pooled data from the PHS I and PHS II, yielding a total of 29071 participants. The return date of the questionnaire containing the RLS questions (216-month questionnaire for PHS I participants and 12-month questionnaire for PHS II participants) was defined as new baseline for this analysis. At this time point, 24505 men were still in active follow-up. We excluded 1579 men with missing information for all three RLS questions, leaving a total of 22926 men at our defined baseline for our analysis.

\section{RLS ASSESSMENT}

RLS is diagnosed according to the presence of specific symptoms. Participants in each study were asked to answer the following questions which were based on the minimal criteria published by the International Restless Legs Syndrome Study Group: ${ }^{12}$ 'Do you have unpleasant leg sensations (like crawling, paraesthesias, or pain) combined with a motor restlessness and an urge to move?', 'Do these symptoms occur only at rest and does moving improve them?', 'Are these symptoms worse in the evening or at night compared with the morning?' The three answer categories included 'Yes', 'No' or 'Don't know'. Participants were only classified as RLS-positive if they answered all symptom questions with 'Yes'.

In DHS and SHIP RLS assessment was conducted in face-to-face interviews by trained and certified interviewers, while in WHS and PHS RLS information was retrieved by mailed standardised questionnaires. The same questions that were used to identify RLS symptoms in each study had been previously validated ${ }^{24}$ and had already been used in prior reports. ${ }^{2} 571825$ Comparing the questionnaire-based classification of RLS with a physician's diagnosis as a gold standard showed good agreement (unweighted $\kappa=0.67, \mathrm{p}<0.001$ ) in the German MEMO (Memory and Morbidity in Augsburg Elderly) study. ${ }^{24}$ In the DHS, there was a further question related to the number of years elapsed since the onset of RLS symptoms. The frequency of RLS symptoms was also assessed in the DHS with the following possible answer categories: 'daily', '3-6 times a week', '1-2 times a week', '1-3 times a month' and 'less than once a month'. The first two categories and the remaining three categories were subsequently collapsed for the analysis.

\section{ASCERTAINMENT OF MORTALITY}

Death from all causes was defined as our main outcome for the analyses. In both German cohorts, the vital status of participants, that is, alive or deceased and the date of death, if applicable, was retrieved from the municipal registries at the follow-up. In both US cohorts, deaths of participants were identified by reports from family members or next of kin, or postal authorities, and searches of the National Death Index. Information on date and cause of death were confirmed through review of death certificates and medical records by an endpoints committee of physicians.

\section{SOCIO-DEMOGRAPHIC DATA, LIFESTYLE FACTORS AND COMORBIDITIES}

Age, gender and lifestyle factors, that is, health-related behaviours, were assessed during the interview in the German studies and with the mailed questionnaire in the WHS and PHS. In the DHS and SHIP, comorbidities including diabetes mellitus, hypertension, cancer, myocardial infarction and stroke were assessed as self-reports with specific questions asking for a physician-made diagnosis of the respective condition. In the WHS and PHS, these comorbidities were also assessed by questionnaires, and the presence of myocardial infarction, stroke and cancer was confirmed by medical record review. Body mass index (BMI) was calculated using measured height and weight information in the German studies and selfreported height and weight information in the US cohorts. There was one question referring to the presence of leg cramps in SHIP.

\section{STATISTICAL ANALYSIS}

Data of the four cohort studies were analysed separately using the following identical analysis approach. For each cohort, we calculated mean values for continuous and frequencies for categorical variables of baseline characteristics. Person-time was calculated from the return date of the questionnaire containing the RLS questions or date of interview, respectively, to the date of death, last documented contact or end of study, whatever occurred first. Cox proportional hazards models were used to 
evaluate the association between RLS status and mortality. Age-adjusted and multivariable-adjusted HRs and their 95\% CIs were calculated. The multivariable models were adjusted for the following potential confounding factors: age, gender (DHS and SHIP), BMI, smoking, exercise, history of diabetes, history of hypertension, myocardial infarction, stroke and cancer. To provide comparability of results across studies, we additionally performed stratified analyses according to gender in the DHS and SHIP.

The proportional hazards assumption was tested by including an interaction term for RLS status and logarithm of follow-up time for mortality in age-adjusted models. We found no statistically significant violation.

In all multivariable models participants with missing covariate information were excluded. The models were reanalysed incorporating a missing value indicator in the outcome models for covariates if the number of participants with missing information was greater or equal to 100. We assigned participants with missing values to the covariate reference category if the number of missing information was less than 100. These analyses provided nearly identical results (data not shown).

We performed several sensitivity analyses. First, we excluded participants who reported leg cramps in SHIP since the presence of leg cramps is a potential RLS mimic. Second, we evaluated whether RLS frequency is associated with RLS mortality in the DHS. Third, we assessed whether the number of years elapsed since the onset of RLS has an effect on RLS mortality in the DHS. All analyses were performed with Stata V.11.0 (StataCorp, Texas, USA) in the DHS and SHIP. For all WHS and PHS analyses, we used SAS V.9.1.3 (SAS Institute Inc, Cary, North Carolina, USA). All $\mathrm{p}$ values were two-tailed and $\mathrm{p}<0.05$ was considered statistically significant.

\section{RESULTS}

\section{Baseline characteristics}

Table 1 summarises the baseline characteristics of participants in the four different cohorts. The mean age in the cohorts ranged from 50.3 years in SHIP to 67.8 years in the PHS. The prevalence of RLS at baseline varied between $7.4 \%$ in DHS and $11.9 \%$ in WHS. Baseline RLS prevalence was significantly higher among women than in men in both German cohorts $(9.3 \%$ vs $5.2 \%, \mathrm{p}=0.005$ in DHS and $12.8 \%$ vs $7.3 \%, \mathrm{p}<0.001$ in SHIP).

\section{RLS and mortality}

The median follow-up time, the number of deaths and the death rate for each study are presented in table 2 . The highest death rate was observed in the PHS (12.3/ 1000 person-years) and the lowest death rate was measured in the WHS (2.8/1000 person-years).

Tables 3 and 4 summarise the age-adjusted and multivariable-adjusted HRs (95\% CI) for the association between RLS and mortality. RLS was not significantly associated with mortality in any of the four studies. Multivariable-adjusted HRs (95\% CI) ranged from 0.21 (0.03 to 1.53) in the DHS to 1.07 (0.93 to 1.23) in the PHS. After gender stratification the adjusted HRs were similar among women and men in SHIP. Furthermore, when the interaction term between RLS and gender was added to the fully adjusted model, it was not significant $(p=0.71)$. In the DHS the estimation of the HR among women was not possible, because no women with RLS died during the follow-up. Excluding participants who reported leg cramps $(n=370)$ did not substantially alter the results in SHIP (multivariable-adjusted HR (95\% CI) 0.85 ( 0.61 to 1.19$)$ ). When the frequency of RLS symptoms was entered into the model in the DHS, the HR associated with the RLS frequency of no more than twice a week versus no RLS symptoms was 0.39 (95\% CI 0.09 to 1.63); the HR for RLS frequency of at least three times a week versus no RLS was 1.28 (95\% CI 0.45 to 3.63). Finally, when the number of years elapsed since the onset of RLS symptoms was added to the multivariable-adjusted model, it was not associated with increased mortality hazard in DHS: HR $=1.00$ (95\% CI 0.90 to 1.12$)$.

\begin{tabular}{lllll}
\multicolumn{5}{l}{ Table 1 Baseline characteristics of participants } \\
\hline & $\begin{array}{l}\text { Dortmund Health } \\
\text { Study }\end{array}$ & $\begin{array}{l}\text { Study of Health } \\
\text { in Pomerania }\end{array}$ & $\begin{array}{l}\text { Physicians' Health } \\
\text { Study }\end{array}$ & $\begin{array}{l}\text { Women's Health } \\
\text { Study }\end{array}$ \\
\hline Number of participants & 1299 & 4291 & 22926 & 31370 \\
Age (years) (mean \pm SD) & $52.2 \pm 13.8$ & $50.3 \pm 16.4$ & $67.8 \pm 9.0$ & $63.6 \pm 6.9$ \\
Women, $\mathrm{n}(\%)$ & $688(53.0)$ & $2185(50.9)$ & $0(0)$ & $31370(100)$ \\
$\mathrm{RLS}, \mathrm{n}(\%)$ & $96(7.4)$ & $433(10.1)$ & $1717(7.5)$ & $3745(11.9)$ \\
$\mathrm{BMI}\left(\mathrm{kg} / \mathrm{m}^{2}\right)($ mean $\pm \mathrm{SD})$ & $27.5 \pm 5.0$ & $27.3 \pm 4.8$ & $25.9 \pm 3.7$ & $27.1 \pm 5.5$ \\
Diabetes, diagnosed, $\mathrm{n}(\%)$ & $98(7.5)$ & $342(8.0)$ & $1983(8.7)$ & $2342(7.5)$ \\
Hypertension, diagnosed, $\mathrm{n}(\%)$ & $461(35.7)$ & $1729(40.8)$ & $12079(52.7)$ & $15223(48.5)$ \\
Cancer, diagnosed, $\mathrm{n}(\%)$ & $59(4.6)$ & $53(1.2)$ & $2306(10.1)$ & $1818(5.8)$ \\
Myocardial infarction, & $49(3.8)$ & $146(3.4)$ & $797(3.5)$ & $248(0.8)$ \\
diagnosed, $\mathrm{n}(\%)$ & & & \\
Stroke, diagnosed, $\mathrm{n}(\%)$ & $29(2.2)$ & $98(2.3)$ & $490(2.1)$ & $251(0.8)$ \\
\hline BMI, body mass index; $\mathrm{RLS}$, restless legs syndrome. & & &
\end{tabular}


Table 2 Follow-up status of participants

\begin{tabular}{|c|c|c|c|c|}
\hline & $\begin{array}{l}\text { Dortmund Health } \\
\text { Study }\end{array}$ & $\begin{array}{l}\text { Study of Health } \\
\text { in Pomerania }\end{array}$ & $\begin{array}{l}\text { Physicians' Health } \\
\text { Study }\end{array}$ & $\begin{array}{l}\text { Women's Health } \\
\text { study }\end{array}$ \\
\hline Number of participants & 1299 & 4291 & 22926 & 31370 \\
\hline Median follow-up time (IQR) & $6.9(0.3)$ & $11.1(1.5)$ & $8.7(0.6)$ & $6.5(0.8)$ \\
\hline \multicolumn{5}{|l|}{ Number of deaths (\%) } \\
\hline Total & $55(4.2)$ & $540(12.6)$ & $2287(10.0)$ & $542(1.7)$ \\
\hline Among participants with RLS & $1(1.0)$ & $70(16.2)$ & $215(12.5)$ & $63(1.7)$ \\
\hline Among participants without RLS & $54(4.5)$ & $470(12.2)$ & $2072(9.8)$ & $479(1.7)$ \\
\hline $\begin{array}{l}\text { Mortality rate per } 1000 \text { person } \\
\text { per year }(95 \% \mathrm{Cl})\end{array}$ & $6.2(4.7$ to 8.1$)$ & $11.8(10.9$ to 12.9$)$ & $12.3(11.8$ to 12.8$)$ & $2.8(2.5-3.0)$ \\
\hline
\end{tabular}

\section{DISCUSSION}

In four independently conducted large prospective cohort studies from Germany and the USA, RLS was not associated with all-cause mortality. The risk of death did not differ according to gender. In contrast to the few previous studies, ${ }^{16} 17$ we applied standardised questions of the minimal diagnostic criteria to assess RLS and we also accounted for numerous explanatory variables.

\section{COMPARISONS WITH OTHER STUDIES}

Some clinic-based studies found a relationship between RLS and mortality among subjects with end-stage renal disease, either in dialysed ${ }^{13} 14$ or transplanted patients. ${ }^{15}$ There was no clear explanation for the findings, although the presence of RLS could be related to indicators of poorer health status, like higher level of uraemia, disease duration and comorbidity. However, those studies were conducted in a very specific patient group with very high morbidity and mortality, and thus the results can be hardly generalised to relatively healthier populations like ours.

In prior reports of community-dwelling elderly subjects, RLS symptoms indicated higher mortality only among women. ${ }^{16}$ Mallon et al found that RLS combined with daytime sleepiness was associated with increased mortality risk among women in a middle-aged population, although RLS without sleepiness was not significantly related to mortality. ${ }^{17}$ There was no clear explanation for these findings, though it has been speculated that disturbed sleep, which frequently accompanies severe RLS, might contribute to the decreased lifespan of subjects with RLS. We had no data about daytime sleepiness or other sleep variables available to determine whether these factors modify the relationship between RLS and mortality.

Differences in sampling and population characteristics might have contributed to the discrepant findings between the previous and the present studies. More importantly, at the time of the baseline assessment of the two earlier studies, standard criteria for RLS did not exist. The urge to move the legs and relief of symptoms due to leg movement, two core symptoms of RLS, were not assessed. It should be also noted that the follow-up time of the study of Mallon et $a l^{17}$ was 20 years, much longer than any of our studies.

In contrast to these previous results, recently published analyses from the WHS and PHS cohorts do not suggest that RLS is associated with incident cardiovascular disease events including CVD mortality, either in women or men. ${ }^{25}$ However, another recent report

Table 3 Age-adjusted, gender-adjusted and multivariable-adjusted HRs and 95\% Cls for mortality according to RLS status in the German cohorts

\begin{tabular}{lll}
\hline & Dortmund Health Study & Study of Health in Pomerania \\
\cline { 2 - 3 } & HR $(95 \% \mathbf{C l})$ & $\mathrm{n}=4291$ \\
\hline Age-adjusted and gender-adjusted models & $\mathrm{n}=1299$ & $1.04(0.81$ to 1.34$)$ \\
Total & $0.21(0.03$ to 1.49$)$ & $1.00(0.71$ to 1.39$)$ \\
Male & $0.42(0.06$ to 3.09$)$ & $1.13(0.77$ to 1.67$)$ \\
Female & $\mathrm{n} . \mathrm{a}$ & $\mathrm{n}=4264$ \\
Multivariable-adjusted* models & $\mathrm{n}=1283$ & $0.99(0.76$ to 1.29$)$ \\
Total & $0.21(0.03$ to 1.53$)$ & $0.98(0.68$ to \\
Male & $0.52(0.07$ to 3.95$)$ & $1.00(0.66$ to 1.50$)$ \\
Female & $\mathrm{NA}$ & \\
\hline
\end{tabular}

*Multivariable models were adjusted for age, gender, body mass index, smoking, physical activity and histories of diabetes, hypertension, myocardial infarction, stroke and cancer.

NA, not available; RLS, restless legs syndrome. 
Table 4 Age-adjusted and multivariable-adjusted HRs and $95 \%$ Cls for mortality according to RLS status in the US cohorts

\begin{tabular}{lll}
\hline & $\begin{array}{l}\text { Women's } \\
\text { Health } \\
\text { Study }\end{array}$ & $\begin{array}{l}\text { Physicians' } \\
\text { Health Study }\end{array}$ \\
\cline { 2 - 3 } & HR (95\% Cl) & \\
\hline Age-adjusted models & $\mathrm{n}=31370$ & $\mathrm{n}=22926$ \\
& 0.98 & 1.10 \\
& $(0.75$ to 1.27$)$ & $(0.96$ to 1.27$)$ \\
Multivariable-adjusted* & $\mathrm{n}=30475$ & $\mathrm{n}=22816$ \\
models & & \\
& 0.93 & 1.07 \\
& $(0.71$ to 1.21$)$ & $(0.93$ to 1.23$)$ \\
\hline
\end{tabular}

*Multivariable models were adjusted for age, body mass index, smoking, physical activity and histories of diabetes, hypertension, myocardial infarction, stroke and cancer.

RLS, restless legs syndrome.

suggests that RLS with a duration at least three years is an independent predictor of coronary heart disease among women. ${ }^{26}$

Subjective and objective sleep disturbances are very common among subjects with RLS. ${ }^{27} 28$ Both short sleep duration and frequent insomnia symptoms predict mortality according to population-based and communitybased studies. ${ }^{29} 30$ Thus we cannot rule out that patients experiencing RLS combined with chronic severe sleep deprivation and/or insomnia may have a higher mortality risk over time. Future prospective studies with assessment of RLS severity as well as various sleep-related factors should further investigate this hypothesis.

\section{STRENGTHS AND LIMITATIONS}

One of the strengths of the present work is the analysis of four independently conducted cohort studies. The lack of association observed across the four different studies indicates the robustness of our finding. Furthermore, RLS cases were classified according to the minimal criteria for RLS. There were differences in the sampling procedures, sample characteristics and baseline data collection across the studies. However, the fact that the results of a null association were the same despite these differences underlines the robustness of the lack of association between RLS and mortality. Finally, we were able to take several important potential confounders into account, including behavioural risk factors and comorbid conditions.

Several limitations have to be considered when interpreting our results. An important limitation is that, as in all large epidemiological studies, case classification was based on a set of self-administered diagnostic questions instead of a complete clinical interview and examination. However, the set of RLS questions used in our cohorts has been successfully used and validated in previous studies and the observed prevalences in our cohorts are similar to those reported in other population-based studies. ${ }^{31}$ We also note that conducting clinical interviews in large studies like these would have been extremely difficult. Still, the use of screening questions could have led to misclassification, and RLS mimics could have been falsely identified as RLS cases. We adjusted for the presence of diabetes, therefore diabetic neuropathy, a common potential RLS mimic, was not likely to influence the findings. In SHIP, excluding participants with leg cramps, another frequent RLS mimic, did not substantially alter the results. Nevertheless, there might be other potential mimics of RLS symptoms that could have an effect on the association between RLS and mortality. Due to the low number of outcome events in the DHS, the estimates for the association of RLS with mortality were less precise than in the other three cohorts. It is possible that a longer follow-up time might have revealed an association between RLS and mortality. Information on frequency and duration of RLS symptoms was available in only one of the cohorts. Despite adjustments for a large number of potential confounders, residual and unmeasureable confounding remains possible as our study is observational. However, we are not aware of any confounding factor that, if included in our final models, would result in increased risk of all-cause mortality among patients with RLS. Participants in all four cohorts were predominately white, which may limit the generalisability to other populations.

\section{CLINICAL IMPLICATIONS}

Results of these four independent large cohort studies do not suggest that RLS is a risk factor for mortality. However, previous studies indicate that RLS is associated with a variety of comorbidities, such as diabetes, higher body mass index and cardiovascular diseases, which are established risk factors for mortality. Patients with RLS should be screened for RLS associated comorbidities and treated if necessary.

\section{UNANSWERED QUESTIONS AND FUTURE RESEARCH}

RLS is a highly prevalent disease among the elderly, but the mechanisms causing the disease and its relationship with a diverse set of comorbidities remain poorly understood. In addition, sleep deprivation and insomnia accompanying RLS may also influence mortality and comorbidity. Further studies are warranted to establish risk factors for incident RLS to be able to develop sufficient prevention strategies and to understand the complex relationship between RLS and its comorbid conditions. In the DHS, the frequency and duration of RLS symptoms were not related to mortality, even though the effect size increased with symptom frequency. Since this was the smallest study with the youngest population, the potential role of RLS severity needs further clarification. Finally, RLS as a potential mortality hazard should be further investigated with longer follow-up time and in 
high-risk populations, for example among elderly subjects.

In summary, results of four independent large, prospective cohort studies indicate that RLS is not associated with increased risk of all-cause mortality. Given the high prevalence of RLS in western populations, this is a positive result for all living with RLS.

\section{Author affiliations}

${ }^{1}$ Institute of Epidemiology and Social Medicine, University of Münster, Münster, Germany

${ }^{2}$ Institute of Behavioural Sciences, Semmelweis University, Budapest, Hungary

${ }^{3}$ Division of Preventive Medicine, Department of Medicine, Brigham and Women's Hospital, Harvard Medical School, Boston, USA

${ }^{4}$ Department of Neurology, University Hospital Essen, Essen, Germany

${ }^{5}$ Institute for Community Medicine, University Medicine Greifswald,

Greifswald, Germany

${ }^{6}$ Centre for Integrated Dementia Care Research (CIDC), German Centre for Neurodegenerative Diseases (DZNE), Greifswald, Germany

${ }^{7}$ Division of Aging, Department of Medicine, Brigham and Women's Hospital, Harvard Medical School, Boston, USA

${ }^{8}$ Department of Medicine, VA Boston Healthcare System, Boston, USA

${ }^{9}$ INSERM Unit 708-Neuroepidemiology, Bordeaux, France

${ }^{10}$ University of Bordeaux, Bordeaux, France.

Acknowledgements We are indebted to all participants for their outstanding commitment and cooperation, to the entire staff of each study for their expert and unfailing assistance.

Contributors AS developed the evaluation plan, made statistical analyses, interpretation of results, literature search and drafted the manuscript. ACW designed the study, developed the evaluation plan, carried out statistical analyses, interpreted the results, performed literature search and drafted the manuscript. HV contributed to data collection, interpretation of results and critically revised the manuscript. WH contributed to data collection, interpretation of results and critically revised the manuscript. MS designed the study, interpreted the results and critically revised the manuscript. JB analysed and interpreted data, obtained funding and critically revised the manuscript. JMG analysed and interpreted data, obtained funding and critically revised the manuscript. TK designed the study, developed the evaluation plan, interpreted the results and contributed to drafting the manuscript. KB designed the study, developed the evaluation plan, made the statistical analysis, interpreted the results and drafted the manuscript.

Funding Data collection in the Dortmund Health Study was supported by the German Migraine \& Headache Society and by unrestricted grants of equal share from Almirall, Astra Zeneca, Berlin Chemie, Boehringer, Boots Health Care, Glaxo-Smith-Kline, Janssen Cilag, McNeil Pharma, MSD Sharp \& Dohme and Pfizer to the University of Muenster.

SHIP is part of the Community Medicine Research Net of the University of Greifswald (available at http://medizin.uni-greifswfald.de/cm) and was funded by grant ZZ9603 from the Federal Ministry of Education and Research, Berlin, and the Ministers of Cultural and Social Affairs of the Federal State of Mecklenburg-West Pomerania, Schwerin.

The RLS research in the WHS and PHS is supported by a grant from the National Heart, Lung, and Blood Institute (HL-091880). The Women's Health Study is supported by grants from the National Heart, Lung, and Blood Institute (HL-043851, HL-080467, HL-099355), and the National Cancer Institute (CA-47988). The Physicians' Health Study is supported by grants from the National Cancer Institute (CA-34944, CA-40360 and CA-097193) and from the National Heart, Lung, and Blood Institute (HL-26490 and HL-34595).

\section{Competing interests None.}

Patient consent Obtained.

Ethics approval All participants in the four cohorts gave informed written consent and the study protocol was approved by the local ethics committees of the Medical Faculty at the University of Münster (for DHS), the University of Greifswald (for SHIP) and the institutional review board of Brigham and Women's Hospital, Boston (for WHS and PHS; Protocol \#: 2008-P-000 613/3).

Provenance and peer review Not commissioned; externally peer reviewed.

Data sharing statement No additional data are available.

\section{REFERENCES}

1. Garcia-Borreguero D, Egatz R, Winkelmann J, et al. Epidemiology of restless legs syndrome: the current status. Sleep Med Rev 2006;10:153-67.

2. Szentkiralyi A, Fendrich K, Hoffmann W, et al. Incidence of restless legs syndrome in two population-based cohort studies in Germany. Sleep Med 2011;12:815-20.

3. Paulus W, Dowling P, Rijsman R, et al. Update of the pathophysiology of the restless-legs-syndrome. Mov Disord 2007;22(Suppl 18): S431-9.

4. Szentkiralyi A, Madarasz CZ, Novak M. Sleep disorders: impact on daytime functioning and quality of life. Expert Rev Pharmacoecon Outcomes Res 2009;9:49-64.

5. Rothdach AJ, Trenkwalder C, Haberstock J, et al. Prevalence and risk factors of RLS in an elderly population: the MEMO study. Memory and Morbidity in Augsburg Elderly. Neurology 2000;54:1064-8.

6. Lee HB, Hening WA, Allen RP, et al. Restless legs syndrome is associated with DSM-IV major depressive disorder and panic disorder in the community. J Neuropsychiatry Clin Neurosci 2008;20:101-5.

7. Berger K, Luedemann J, Trenkwalder C, et al. Sex and the risk of restless legs syndrome in the general population. Arch Intern Med 2004;164:196-202

8. Ulfberg J, Nystrom B, Carter N, et al. Prevalence of restless legs syndrome among men aged 18 to 64 years: an association with somatic disease and neuropsychiatric symptoms. Mov Disord 2001;16:1159-63.

9. Walters AS, Moussouttas M, Siddiqui F, et al. Prevalence of stroke in Restless Legs Syndrome: initial results point to the need for more sophisticated studies. Open Neurol J 2010;4:73-7.

10. Winkelman JW, Shahar E, Sharief I, et al. Association of restless legs syndrome and cardiovascular disease in the Sleep Heart Health Study. Neurology 2008;70:35-42.

11. Ohayon MM, Roth T. Prevalence of restless legs syndrome and periodic limb movement disorder in the general population. $J$ Psychosom Res 2002;53:547-54.

12. Walters AS. Toward a better definition of the restless legs syndrome. The International Restless Legs Syndrome Study Group. Mov Disord 1995;10:634-42.

13. La Manna G, Pizza F, Persici E, et al. Restless legs syndrome enhances cardiovascular risk and mortality in patients with end-stage kidney disease undergoing long-term haemodialysis treatment. Nephrol Dial Transplant 2011;26:1976-83.

14. Unruh ML, Levey AS, D'Ambrosio C, et al. Restless legs symptoms among incident dialysis patients: association with lower quality of life and shorter survival. Am J Kidney Dis 2004;43:900-9.

15. Molnar MZ, Szentkiralyi A, Lindner A, et al. Restless legs syndrome and mortality in kidney transplant recipients. Am J Kidney Dis 2007:50:813-20.

16. Pollak CP, Perlick D, Linsner JP, et al. Sleep problems in the community elderly as predictors of death and nursing home placement. J Community Health 1990;15:123-35.

17. Mallon L, Broman JE, Hetta J. Restless legs symptoms with sleepiness in relation to mortality: 20-year follow-up study of a middle-aged Swedish population. Psychiatry Clin Neurosci 2008;62:457-63.

18. Happe S, Vennemann M, Evers S, et al. Treatment wish of individuals with known and unknown restless legs syndrome in the community. J Neurol 2008;255:1365-71.

19. Volzke H, Alte D, Schmidt CO, et al. Cohort profile: the study of health in Pomerania. Int J Epidemiol 2011;40:294-307.

20. Rexrode KM, Lee IM, Cook NR, et al. Baseline characteristics of participants in the Women's Health Study. J Womens Health Gend Based Med 2000;9:19-27.

21. Ridker PM, Cook NR, Lee IM, et al. A randomized trial of low-dose aspirin in the primary prevention of cardiovascular disease in women. N Engl J Med 2005;352:1293-304.

22. Steering Committee of the Physicians' Health Study Research Group. Final report on the aspirin component of the ongoing Physicians' Health Study. N Engl J Med 1989;321:129-35. 
23. Christen WG, Gaziano JM, Hennekens CH. Design of Physicians' Health Study II-a randomized trial of beta-carotene, vitamins $E$ and C and multivitamins, in prevention of cancer, cardiovascular disease, and eye disease, and review of results of completed trials. Ann Epidemiol 2000;10:125-34.

24. Berger $\mathrm{K}$, von Eckardstein A, Trenkwalder $\mathrm{C}$, et al. Iron metabolism and the risk of restless legs syndrome in an elderly general population-the MEMO-Study. J Neurol 2002;249:1195-9.

25. Winter AC, Schurks M, Glynn RJ, et al. Restless legs syndrome and risk of incident cardiovascular disease in women and men: prospective cohort study. BMJ Open 2012;2:e000866.

26. LI Y, Walters AS, Chiuve SE, et al. Prospective study of restless legs syndrome and coronary heart disease among women. Circulation 2012;126:1689-94.

27. Hening W, Walters AS, Allen RP, et al. Impact, diagnosis and treatment of restless legs syndrome (RLS) in a primary care population: the REST (RLS epidemiology, symptoms, and treatment) primary care study. Sleep Med 2004;5:237-46.

28. Montplaisir J, Boucher S, Poirier G, et al. Clinical

polysomnographic, and genetic characteristics of restless legs syndrome: a study of 133 patients diagnosed with new standard criteria. Mov Disord 1997;12:61-5.

29. Cappuccio FP, D'Elia L, Strazzullo P, et al. Sleep duration and all-cause mortality: a systematic review and meta-analysis of prospective studies. Sleep 2010;33:585-92.

30. Chien $\mathrm{KL}$, Chen PC, Hsu HC, et al. Habitual sleep duration and insomnia and the risk of cardiovascular events and all-cause death: report from a community-based cohort. Sleep 2010;33:177-84.

31. Berger K, Kurth T. RLS epidemiology - frequencies, risk factors and methods in population studies. Mov Disord 2007;22(Suppl 18): S420-3. 